\title{
The Effect of the Exciton Continuum on the Exciton-Exciton Correlation
}

\author{
G. Martino, S. Savasta, and R. Girlanda ${ }^{1}$ ) \\ INFM and Dipartimento di Fisica della Materia e Tecnologie Fisiche Avanzate, \\ Università di Messina, Salita Sperone 31, I-98166 Messina, Italy
}

\begin{abstract}
We present a numeric calculation of the exciton-exciton correlation for a one-dimensional semiconductor model with long-range Coulomb interactions, taking into account the effect of the exciton continuum. We use the obtained correlation functions to calculate the time integrated intensity of the four wave mixing signal. Changing the central frequency of the exciting pulses we observe changes in the profile and in the rise time of the time integrated signal for negative delays in qualitative agreement with experimental results. Our results show theoretically how the exciton continuum affects the coherence of four-particle correlations.
\end{abstract}

Transient four wave mixing (FWM) experiments in semiconductors have shown the importance of biexciton effects as well as of two-exciton scattering states. In this respect transient FWM has been shown to be a versatile tool for investigating four-particle Coulomb correlations. Direct investigations of the coherence of four-particle correlations in semiconductors have been carried out by ultrafast FWM experiments [1]. The signature of the exciton-exciton correlation in FWM has stimulated much theoretical effort to improve the mean-field theory of the semiconductor Bloch equations. Within the $\chi^{3}$ truncation scheme, the theory of coherent FWM can be expressed in terms of two types of correlation functions: the two-particle correlations and the four-particle correlations [2]. These functions obey a set of coupled differential equations which can be solved numerically. Östreich et al. have presented a theory of exciton-exciton interaction effects in the third-order nonlinear response using a correlation function approach [3]. They calculated numerically the correlation function for the lowest exciton level (1s) for a one-dimensional semiconductor model with long-range Coulomb interaction without perturbative approximation. The optical response near the band edge in direct gap semiconductors is dominated by 1s excitons, however, the large spectral profile of the ultrafast exciting pulses determines the excitation of 1 s excitons as well as of the exciton continuum. In particular changing the central frequency of the laser one changes the relative number of $1 \mathrm{~s}$ excitons and $\mathrm{e}-\mathrm{h}$ pairs of the continuum generated by the input beam. Time integrated (TI) FWM experiments reveal that the detuning has a much greater effect on the coherence of four-particle correlations rather than on the coherence of two-particle correlations (excitons) [1].

In order to investigate theoretically the effect of the exciton continuum on the exciton-exciton correlation, we have calculated numerically the $\chi^{3}$ nonlinear response for a one-dimensional semiconductor model with long-range Coulomb interaction by consid-

1) e-mail: Raffaello.Girlanda@unime.it 
ering two- and four-particle correlations where states from the $\mathrm{e}-\mathrm{h}$ continuum are also included. Here we present results for cross polarized circular excitation. In the following we will avoid indicating explicitly the polarization state of input light and of the semiconductor excitations.

The third-order nonlinear polarization can be expressed as a summation over all the exciton states which we label by the quantum number $n$ as $P^{3}(t)=\sum_{n} P_{n}^{3}(t) . P_{n}^{3}(t)$ in the coherent limit and for cross polarized excitations is given by the solution of the following linear differential equation with a source term [3]:

$$
\left(\frac{\partial}{\partial t}+i \omega_{n}+\Gamma\right) P_{n}^{3}(t)=-\frac{i}{2} M_{n}^{*}\left[X_{\tilde{n}}(t)\right]^{*} \int_{-\infty}^{t} \mathrm{~d} t^{\prime} \mathrm{e}^{-2 \Gamma\left(t-t^{\prime}\right)} F_{\tilde{n}, n}^{n^{\prime}, n^{\prime \prime}}\left(t-t^{\prime}\right) X_{n^{\prime}}\left(t^{\prime}\right) X_{n^{\prime \prime}}\left(t^{\prime}\right) .
$$

In this equation $\omega_{n}$ and $M_{n}^{*}$ are respectively the energy and the dipole moment of the $n$-th exciton level, $F_{\tilde{n}, n}^{n^{\prime}, n^{\prime \prime}}\left(t-t^{\prime}\right)$ is the exact exciton-exciton correlation function in the $\chi^{(3)}$ limit. $\Gamma$ describes the exciton dephasing, and the functions $X_{n}(t)$ are linear exciton amplitudes obeying the following differential equations:

$$
\left(\frac{\partial}{\partial t}+i \omega_{n}+\Gamma\right) X_{n}(t)=\frac{i}{2} M_{n} E(t)
$$

where $E(t)$ is the strength of the exciting field. The exact calculation of the excitonexciton correlation function is tightly bound to the solution of the four-particle problem. The calculation of the correlation functions is extremely time consuming; if one has to include in the calculation $N$ exciton levels, one has to calculate $N^{4}$ different correlation functions. We have calculated numerically the correlation functions for a one-dimensional semiconductor with long-range Coulomb interaction and without perturbative approximations in the Coulomb interaction. Here we present the results including the contribution from the bound exciton and the first most relevant contributions from the continuum to the FWM response.

By inspecting the above equations, determining the evolution of the third-order nonlinear polarization, it results that the contribution to $P^{(3)}(t)$ of the different exciton levels is related to the terms $M_{n} M_{\tilde{n}} M_{n^{\prime}} M_{n^{\prime \prime}} F_{\tilde{n}, n}^{n^{\prime}, n^{\prime \prime}}\left(t-t^{\prime}\right)$. We point out that for one-dimensional semiconductor systems the dipole moments of the exciton continuum are much smaller than the dipole moment of the 1 s exciton $(n=1)$ [4]. As a consequence the main contribution comes from the term $\left(M_{1}\right)^{4} F_{1,1}^{1,1}\left(t-t^{\prime}\right)$. The next relevant contributing terms are those in which three $n=1$ exciton levels are involved and these are the first terms involving contributions from the exciton continuum. An analogous scheme has been adopted for the calculation of the most relevant contributions to the bleaching of the $1 \mathrm{~s}$ exciton resonance [5]. In order to calculate the exciton-exciton correlation functions we use a tight-binding one-dimensional semiconductor model including a long-range Coulomb interaction. The Hamiltonian $H=H_{0}+V$ is given by

$$
H_{0}=E_{\mathrm{g}} \sum_{m} c_{m}^{\dagger} c_{m}+\left(t^{(\mathrm{e})} \sum_{m} c_{m}^{\dagger} c_{m+1}+t^{(\mathrm{h})} \sum_{m} d_{m}^{\dagger} d_{m+1}+\text { h.c. }\right)
$$

with the electrostatic Coulomb interaction

$$
V=\frac{1}{2} \sum_{m, m^{\prime}} U_{m, m^{\prime}} \varrho_{m} \varrho_{m^{\prime}}
$$


with the charge density $\varrho_{m}=c_{m}^{\dagger} c_{m}-d_{m}^{\dagger} d_{m}$. In the above expressions $c_{m}$ and $d_{m}$ are respectively the annihilation operators for electrons in the conduction band and holes in the valence band. For the Coulomb interaction we use

$$
U_{m, m^{\prime}}=U \frac{\pi}{N_{\mathrm{s}} \sin \left(\pi\left|m-m^{\prime}\right| / N_{\mathrm{s}}\right)},
$$

$N_{\mathrm{s}}$ being the number of sites in the one-dimensional semiconductor ring, for $m \neq m^{\prime}$; the on-site Coulomb interaction is a different parameter $U_{m, m}=\eta U$. In particular we have calculated the correlation function in the frequency domain and we obtained $F_{\tilde{n}, n}^{n^{\prime}, n^{\prime \prime}}(\tau)$ after Fourier transform. The correlation function in the frequency domain can be written as [6]

$$
\tilde{F}_{\tilde{n}, n}^{n^{\prime}, n^{\prime \prime}}(\omega)=\left\langle 0\left|D_{\tilde{n}, n} \frac{1}{\omega-H+i 0} D_{n^{\prime}, n^{\prime \prime}}^{\dagger}\right| 0\right\rangle,
$$

where $H$ is the semiconductor Hamiltonian and $D_{\tilde{n}, n}=\left[B_{\tilde{n}},\left[B_{n}, H\right]\right]$ with $B_{n}$ being the annihilation operator of the $n$-th exciton. For each correlation function the total number of basis states involved in the calculation is $N_{\mathrm{s}}^{3}$. We use the Lanczos algorithm to tridiagonalize the Hamiltonian of the four-particle problem. The inversion of the resolvent matrix is quite simple in the tridiagonal representation of $H$. In Fig. 1 we display the spectrum of the correlation function $\tilde{F}_{1,1}^{1,1}(\omega)$, obtained for a semiconductor ring of size $N_{\mathrm{s}}=250$. We have used the following parameters: $t^{\mathrm{e}}=-0.230 \mathrm{eV}, t^{\mathrm{h}}=0.0343 \mathrm{eV}$, $U=0.03 \mathrm{meV}$ and $\eta=1.5$. The other relevant calculated correlation functions present a very close behaviour as a function of energy, but a different lower intensity depending on the exciton level $n$.

Once determined the correlation functions we integrate Eq. (1) by considering a three-pulse excitation scheme, where two pulses of opposite helicity interact with the sample at the same time $\left(t_{2}=t_{3}=0\right)$ and a probe pulse interacts with the sample at time $t_{1}=-T$ ( $T$ is the delay time). The frequency profiles of the laser pulses which we used are displayed in the inset of Fig. 2. We have calculated the time integrated FWM

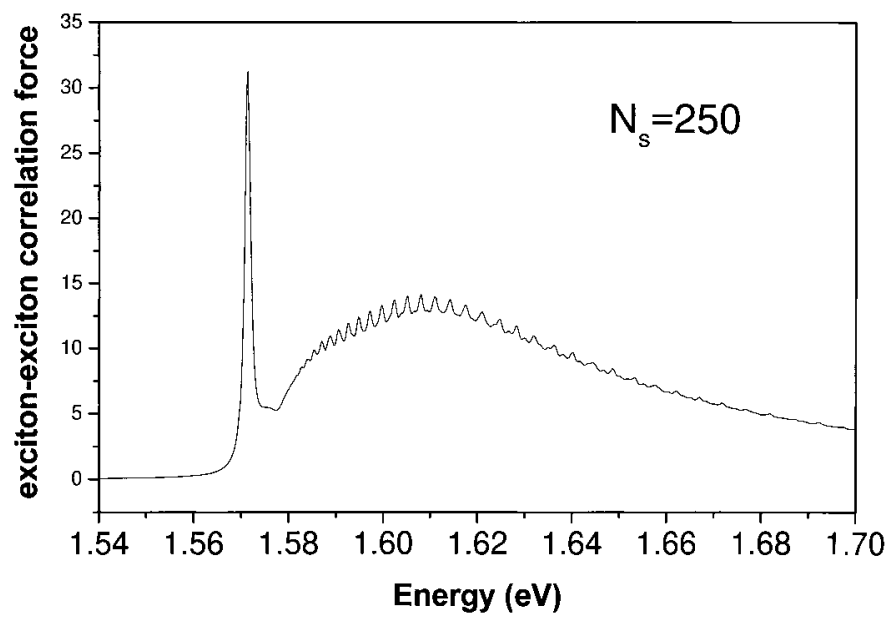

Fig. 1. Spectrum of the exciton-exciton correlation function for the 1s exciton contribution for opposite spins 


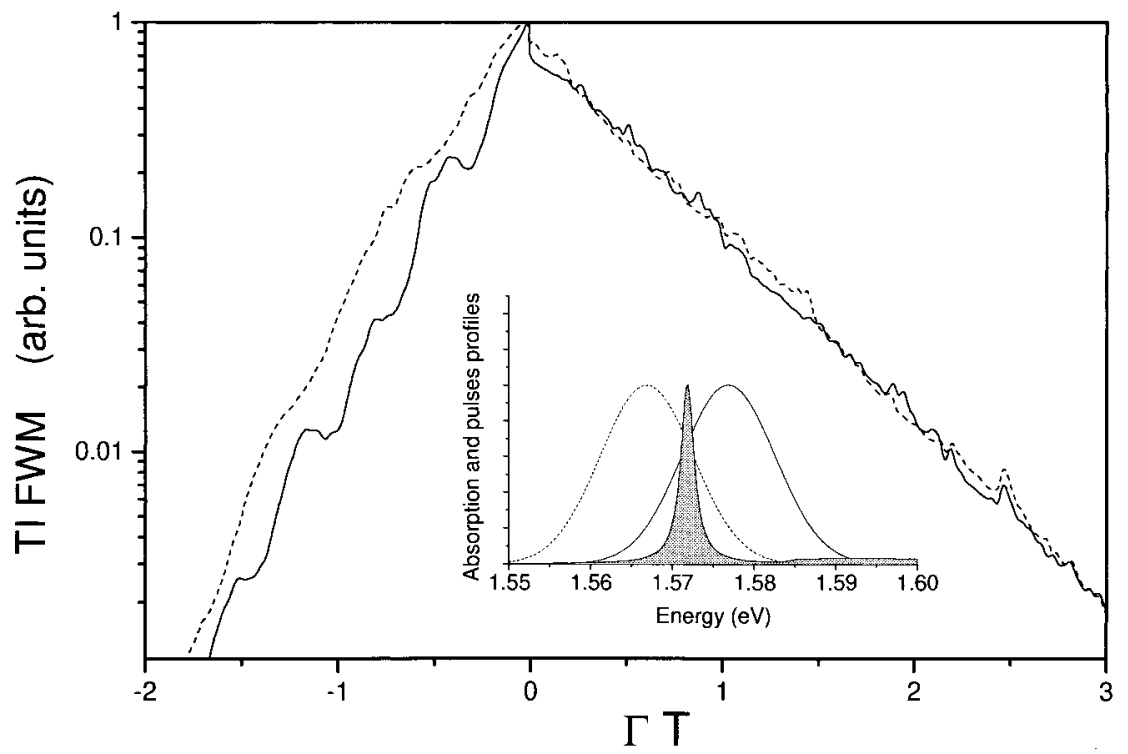

Fig. 2. Calculated time integrated four wave mixing signal as a function of the time delay $T$. The dashed curve is the TI FWM obtained with the central frequency of the laser beams shifted by $\Delta_{-}=-5 \mathrm{meV}$ with respect to the absorption peak of the bound exciton, while the continuous curve is the TI FWM obtained with the central frequency of the laser beams shifted by $\Delta_{+}=5 \mathrm{meV}$. The inset displays the two laser spectra superimposed on the absorption profile of the one-dimensional semiconductor model

signal $I^{(3)}(T)$ as a function of the delay time $T$

$$
I^{(3)}(T)=\int_{\infty}^{\infty} \mathrm{d} t\left|P^{(3)}(t, T)\right|^{2} .
$$

In particular the dashed curve in Fig. 2 is the TI FWM obtained with the central frequency of the laser beams shifted by $\Delta_{-}=-5 \mathrm{meV}$ with respect to the absorption peak of the bound exciton, while the continuous curve is the TI FWM obtained with the central frequency of the laser beams shifted by $\Delta_{+}=5 \mathrm{meV}$. The inset displays the two laser spectra superimposed on the linear absorption profile of the one-dimensional semiconductor model which we used to perform the numerical calculations. The absorption spectrum displayed in the inset in Fig. 2 is defined in the usual way as

$$
f(\omega)=2 \Gamma \operatorname{Im}\left\langle P\left|\frac{1}{\omega+i \Gamma-H}\right| P^{\dagger}\right\rangle,
$$

with $\left|P^{\dagger}\right\rangle=\sum_{m} c_{m}^{\dagger} c_{m}|0\rangle$. The calculation of the linear absorption spectrum has been performed by using the Lanczos algorithm as done for the calculation of the excitonexciton correlation functions and using the same parameters specified above. The exciton dephasing rate has been chosen to be $\Gamma=1 \mathrm{meV}$. The absorption spectrum clearly displays a large peak originating from the lowest exciton level $(n=1)$ and a background at higher energies due to the exciton continuum.

Figure 2 displays a different rise time $(T<0)$ in the TI FWM signal for the two different central frequencies of the laser displayed in the inset of Fig. 2. On the con- 
trary the decay time profile $(T>0)$ does not present significant modifications in the two curves. These two different behaviours for negative and positive decay times are in qualitative agreement with the experimental results [1]. It is known [7] that the signal at negative delay is completely dominated by the four-particle correlations, while at positive delay the decay of the TI FWM signal is determined by the excitonic dephasing rate and hence by the decoherence of two-particle correlations. Our results clearly indicate that the inclusion of the exciton continuum and the changing of the central frequency of the laser affect the coherence of four-particle correlations and have little consequences on the coherence of two-particle correlations. The results here presented are not intended to quantitatively describe the experimental results in quantum wells. In particular we point out that in one-dimensional and quasi-one-dimensional semiconductor systems the contribution to the optical density of states and hence to the absorption of the exciton continuum is very small with respect to the contribution of the lowest $1 \mathrm{~s}$ exciton level [4] as can be seen looking at the absorption profile displayed in the inset of Fig. 2. The contribution of the exciton continuum is largely more important in quasi-two-dimensional systems. The numerical calculation of all the correlation functions and their inclusion in the calculation of the time resolved and time integrated FWM response is under current development.

\section{References}

[1] P. KNeR et al., Phys. Rev. Lett. 81, 5386 (1998).

[2] K. VICtOR et al., Phys. Rev. B 51, 14164 (1995).

[3] Th. Östreich et al., Phys. Rev. Lett. 74, 4698 (1995).

[4] F. Rossi and E. Molinari, Phys. Rev. Lett. 76, 3642 (1996).

[5] S. Schmitt-Rink et al., Phys. Rev. B 32, 6601 (1985).

[6] Th. Östreich et al., Phys. Rev. B 58, 12920 (1998).

[7] P. KNER et al., Phys. Rev. Lett. 78, 1319 (1997). 